\title{
Características Psicométricas de la Escala de Funcionamiento Ejecutivo para Familias (EFE-F)
}

\section{Psychometric Characteristics of the Executive Functioning Scale for Families (EFS-F)}

\author{
Trinidad García ${ }^{1,3}$, David Álvarez-García ${ }^{1}$, Débora Areces ${ }^{1}$, Luis Antonio Segurola ${ }^{2}$, Paloma \\ González-Castro ${ }^{1}$ y Celestino Rodríguez ${ }^{1}$
}

\section{Resumen}

El objetivo de este trabajo es presentar las propiedades psicométricas de un cuestionario breve para la evaluación del funcionamiento ejecutivo en niños y adolescentes, que adopta la forma de escala de valoración de la conducta, a cumplimentar por la familia. Tras una validación piloto previa, la Escala de Funcionamiento Ejecutivo para Familias (EFE-F) fue puesta a prueba con las familias de 1019 estudiantes de 8 a 18 años de edad, pertenecientes a 41 centros educativos de Asturias (España). Los análisis factoriales confirmatorios mostraron que el modelo que mejor se ajusta a los datos es el formado por nueve factores: Inhibición, Hiperactividad, Control Emocional, Capacidad de Concentración, Focalización de la Atención, Capacidad de Planificación, Organización, Memoria Funcional y Flexibilidad. La fiabilidad fue elevada para el conjunto de la escala y moderada para los factores que la componen.

Palabras clave: evaluación, funciones ejecutivas, familia, infancia, adolescencia

\begin{abstract}
The aim of this study is to present the psychometric properties of a brief questionnaire for the assessment of executive functioning in children and adolescents, which takes the form of a behavior rating scale, and designed to be answered by families. After a validation pilot study, the Executive Functioning Scale for Families (EFE-F in Spanish) was tested with the families of 1019 students aged 8-18, from 41 schools in Asturias (Spain). Confirmatory factor analyses showed that the model that best fits the data is composed by nine factors: Inhibition, Hyperactivity, Emotional Control, Concentration, Focus, Planning, Organization, Functional Memory and Flexibility. Reliability was high for the whole scale and moderate for its factors.
\end{abstract}

Keywords: assessment, executive functions, family, childhood, adolescence

Agradecimientos: Este trabajo fue realizado con el apoyo de un proyecto concedido por el Principado de Asturias (FC-15GRUPIN14-053) y el Programa Severo Ochoa de Ayudas Predoctorales-Ficyt (BP14-030).

\footnotetext{
${ }^{1}$ Departamento de Psicología, Universidad de Oviedo, España.

${ }^{2}$ Consejería de Educación y Cultura del Principado de Asturias, España.

${ }^{3}$ Autora para la correspondencia: Plaza Feijoo s/n, 33003- Oviedo, España. Tel.: +34985103259. Correo: garciatrinidad@uniovi.es
} 


\section{Introducción}

En los últimos años, se ha destacado el papel del funcionamiento ejecutivo en el comportamiento humano. Por funcionamiento ejecutivo se entiende un conjunto de procesos cognitivos implicados en el inicio, la supervisión, la regulación, la ejecución y el reajuste de la conducta para alcanzar un determinado objetivo, especialmente en situaciones que requieren un abordaje novedoso y creativo (Lezak, 2004; Verdejo-García \& Bechara, 2010). Estudios neuroanatómicos (Lozano \& Ostrosky, 2011; Tirapú-Ustárroz, García, Luna, Roig, \& Pelegrín, 2008), psicométricos (Egeland \& Fallmyr, 2010; Huizinga \& Smidts, 2011) y evolutivos (Korzeniowski, 2011; Roselli, Jurado, \& Matute, 2008) han apoyado la idea de que el funcionamiento ejecutivo no es una entidad unitaria, sino compuesta por diferentes capacidades o funciones, entre las que destacan: a) la inhibición de respuestas, o capacidad para resistir un impulso, la cual abarca tanto aspectos conductuales o de actividad motora (hiperactividad) como cognitivos (impulsividad); b) la atención, referida tanto a la capacidad para seleccionar los estímulos relevantes y darles prioridad (focalización de la atención), como para concentrarse durante un largo tiempo en una actividad (concentración); c) el control emocional, o capacidad para tomar las riendas de los sentimientos negativos, como el enfado o la ansiedad; d) la memoria funcional o memoria de trabajo, que permite mantener activada una cantidad limitada de información mientras esta se lleva a cabo una actividad; e) la planificación, o capacidad para elaborar un plan orientado a un objetivo; f) la organización, que permite establecer un orden tanto en aspectos físicos como en la información manejada; y g) la flexibilidad cognitiva, o capacidad para adecuar la conducta a las demandas cambiantes del contexto, así como aceptar otros puntos de vista o alternativas en la resolución de un problema.

\section{Modelos de funcionamiento ejecutivo}

El cuestionario elaborado en este estudio parte de una caracterización multidimensional de las funciones ejecutivas, definiendo éstas como un conjunto de componentes de control independientes, pero interrelacionados entre sí. Esta estructura tiene sus bases en modelos actuales como el de Baddeley (2000, 2007), Miyake et al., (2000) o Diamond (2013), los cuales se describen brevemente a continuación.

Baddeley (2000, 2007) distingue entre tres componentes principales, que son la memoria de trabajo y la actualización de la información pertinente ("updating"), estrechamente relacionada con el mantenimiento de la atención; la inhibición de impulsos prepotentes o automáticas ("inhibition"); y el cambio o flexibilidad mental ("shifting"), entendido como la capacidad para cambiar el foco de atención entre diferentes tareas. Tanto el foco como el sostenimiento de la atención son aspectos esenciales de este modelo.

En esta misma línea, Diamond (2013), define las funciones ejecutivas como "funciones de control ejecutivo o control cognitivo" (pp. 136). Basándose en el modelo de Miyake et al. (2000), Diamond destaca tres componentes básicos dentro de las funciones ejecutivas: la inhibición o control inhibitorio, incluyendo dentro de esta función el auto-control (inhibición conductual) y el control de la interferencia (atención selectiva e inhibición cognitiva); la memoria de trabajo; y la flexibilidad cognitiva. Del mismo modo, la autora destaca la existencia de otros componentes de orden superior, concretamente el razonamiento, la resolución de problemas y la planificación (Collins \& Koechlin, 2012). Todas estas funciones serían esenciales para la salud física y mental del individuo, el éxito escolar y/o laboral, así como para su desarrollo cognitivo, emocional y psicológico.

Finalmente, y en la línea de los modelos multidimensionales de funcionamiento ejecutivo previamente expuestos, es de destacar el modelo de Gioia, Isquith, Guy y Kenworthy (2000), creadores de la escala Behavior Rating Inventory of Executive Functions (BRIEF). Partiendo del conocido modelo de Autorregulación de Barkley (1997, 1998), estos autores señalan como componentes a evaluar los siguientes: inhibición, flexibilidad o cambio, control emocional, iniciativa, memoria de trabajo, organización, planificación, y monitorización (Gioia et al., 2000). Este modelo es importante porque tiene en cuenta un componente de control emocional en el 
funcionamiento ejecutivo, aspecto que, si bien está implícito en el resto de teorías, solamente adquiere entidad propia en este último caso.

\section{Relevancia de las funciones ejecutivas}

Aunque las diferentes funciones ejecutivas se desarrollan a diferentes ritmos, existe un amplio consenso en considerar la niñez y la adolescencia etapas especialmente cruciales para su desarrollo (Lozano \& Ostrosky, 2011; Roselli et al., 2008). De ahí la importancia de contar con buenos instrumentos diagnósticos y con buenos métodos de intervención para analizar y optimizar el funcionamiento ejecutivo en estas edades. Alteraciones en estas funciones durante estas edades se traducen en dificultades a nivel cognitivo, conductual y emocional. Así, un inadecuado funcionamiento ejecutivo se ha relacionado con un bajo rendimiento académico y limitaciones en el razonamiento matemático, la lectura y la escritura (Cragg \& Gilmore, 2014; García, Rodríguez, González-Castro, Álvarez, Cueli, \& González-Pienda, 2013; García, Kroesbergen, Rodríguez, González-Castro, \& Gonzalez-Pienda, 2015; Mayoral, Roca, Timoneda, \& Serra, 2015) o con problemas conductuales y de adaptación al contexto social (Brock, Rimm-Kaufman, Nathanson y Grimm, 2009; Garon, Bryson, \& Smith, 2008); y se ha empleado en el diagnóstico diferencial de numerosos desórdenes, como el Trastorno por Déficit de Atención con Hiperactividad-TDAH, los Trastornos del Espectro Autista y otras condiciones clínicas (Di Trani et al., 2011; García, González-Pienda, Rodríguez, Álvarez-García, \& Álvarez, 2014; Leung, Vogan, Powell, Anagnostou, \& Taylor, 2016; Shimoni, EngelYeger, \& Tirosh, 2012).

\section{Evaluación de los componentes de las funciones ejecutivas}

Las pruebas psicológicas empleadas en la evaluación del funcionamiento ejecutivo se pueden clasificar en dos grandes grupos. Por un lado, se encuentran las medidas basadas en la ejecución, que incluyen las pruebas individuales por componentes y las baterías ejecutivas. Entre las pruebas individuales por componentes, destacan las pruebas Stroop Test (Stroop, 1935; Golden, 1974; Musso, 2009) para la evaluación de la interferencia e inhibición de respuestas; Wisconsin Card Sorting Test (WCST; Heaton, Chelune, Talley, Kay y Curtis, 1993) para evaluar la flexibilidad cognitiva; y Tower of Hanoi (ToH; Borys, Spitz y Dorans, 1982) y sus variantes como medida de la planificación. Entre las baterías ejecutivas, destacan el Delis-Kaplan Executive Function System (D-KEFS; Delis, Kaplan y Kramer, 2001), el Behavioral Assessment of the Dysexecutive Syndrome for Children (BADS-C; Kobayashi y Kobayashi, 2007) y la batería de Evaluación Neuropsicológica de las Funciones Ejecutivas (ENFEN; Portellano, Martínez-Arias \& Zumárraga, 2009). Estas medidas neuropsicológicas basadas en la ejecución son extensamente empleadas, y han demostrado su utilidad en la identificación de diversos grupos clínicos, entre ellos el Trastorno por Déficit de Atención con Hiperactividad-TDAH o los Trastornos del Espectro Autista (Craig et al., 2016; Holmes et al., 2010). Una de las dificultades en este sentido es el hecho de que se les atribuye, en ocasiones, una baja validez ecológica (Lezak, Howieson, Bigler, \& Tranel, 2012; Verdejo-García \& Bechara, 2010). De ahí la necesidad de disponer de herramientas de evaluación complementarias.

Un complemento al anterior tipo de medidas es el uso de cuestionarios de calificación de la conducta. Este tipo de instrumentos permite recabar información acerca de las conductas exhibidas por el evaluado en el hogar o el centro educativo. Entre los posibles informantes de las conductas de un niño o adolescente, que permitan conocer su funcionamiento ejecutivo, destaca la familia (García et al., 2015; Huizinga \& Smidts, 2011; Egeland \& Fallmyr, 2010). Los instrumentos de evaluación estandarizados de este tipo más conocidos son el Children's Executive Functions Scale (CEFS; Silver, Kolitz-Russell, Bordoni, \& Fairbanks, 1993; Goulden \& Silver, 2009), el Children Executive Function Inventory (CHEXI; Thorell \& Nyberg, 2008) y la escala BRIEF (Gioia et al., 2000). Si bien estas pruebas han mostrado su utilidad, solamente la última ha sido traducida al español. Una de las limitaciones de este tipo de escalas es su longitud, ya que a menudo están compuestas por un gran número de ítems. Esto impone ciertas restricciones a la hora de llevar a cabo estudios con muestras amplias, 
sobre todo en aquellos casos en que se requiere administrar varias pruebas o cuestionarios.

En definitiva, si bien se dispone de numerosos instrumentos para la evaluación del funcionamiento ejecutivo en niños y adolescentes, la necesidad de medidas complementarias a las pruebas de ejecución tradicionalmente empleadas, la escasa presencia de herramientas adaptadas a nuestro contexto lingüístico y cultural (Elosua, Mujika, Almeida, \& Hermosilla, 2014), o ciertas características de las medidas existentes, sugieren la necesidad de seguir trabajando en el desarrollo de instrumentos de evaluación con ese fin. El objetivo del presente trabajo es presentar las propiedades psicométricas de un cuestionario breve de calificación de conducta para la evaluación del funcionamiento ejecutivo en niños y adolescentes de 8 a 18 años, tras su aplicación a una amplia muestra de familias de Asturias (España).

\section{Método}

\section{Participantes}

Se utilizaron dos muestras: una piloto, con la que se realizaron análisis factoriales exploratorios; y otra final, con la que se realizaron análisis factoriales confirmatorios y de fiabilidad. La muestra piloto está compuesta por las familias de 209 estudiantes d e tercer ciclo de Educación
Primaria (cursos $5^{\circ}$ y $6^{\circ}$ ), pertenecientes a dos centros educativos de Asturias (España). La muestra final está compuesta por las familias de 1019 estudiantes que se encuentran cursando entre segundo ciclo de Educación Primaria y segundo ciclo de Educación Secundaria Obligatoria (ESO), pertenecientes a 41 centros educativos de Asturias. En ambos casos, la muestra fue seleccionada mediante muestreo de conveniencia (Martínez, 1995). En la Tabla 1 se ofrecen los datos descriptivos de ambas muestras.

\section{Instrumento de evaluación}

El instrumento de evaluación puesto a prueba en este estudio es la Escala de Funcionamiento Ejecutivo para Familias (Escala EFE-F). Se trata de una escala breve tipo Likert, compuesta por 27 ítems con 5 opciones de respuesta (desde $1=$ Nunca, hasta 5 = Siempre). El informante (padre, madre o tutor) debe indicar la frecuencia con la que el niño o adolescente muestra una serie de conductas, indicativas de posibles déficits en las funciones ejecutivas. Como en otros instrumentos previamente publicados (e.g. escala BRIEF, de Gioa et al., 2000), la redacción de los diferentes ítems hace referencia tanto a situaciones académicas, como familiares y sociales en las que normalmente están involucrados niños y adolescentes (véase Tabla 2).

Tabla 1. Datos descriptivos de las muestras piloto y final

\begin{tabular}{llcc}
\hline & & Muestra piloto & Muestra final \\
\hline Informante & Madre & $54.5 \%$ & $68.9 \%$ \\
& Padre & $9.1 \%$ & $14.0 \%$ \\
Madre y padre & $35.4 \%$ & $15.2 \%$ \\
Sexo del alumno & Tutor & $1.0 \%$ & $1.9 \%$ \\
& & & \\
Curso del alumno & Alumno & $50.2 \%$ & $53.1 \%$ \\
& Alumna & $49.8 \%$ & $46.9 \%$ \\
& & & $4.1 \%$ \\
& $3^{\circ}$ EP & - & $7.6 \%$ \\
& $4^{\circ}$ EP & - & $25.6 \%$ \\
$5^{\circ}$ EP & 44.0 & $28.4 \%$ \\
& $6^{\circ}$ EP & 56.0 & $11.1 \%$ \\
Edad del alumno & $1^{\circ}$ ESO & - & $7.7 \%$ \\
& $2^{\circ}$ ESO & - & $8.7 \%$ \\
& $3^{\circ}$ ESO & - & $6.7 \%$ \\
TOTAL & $4^{\circ}$ ESO & - & $7-18$ años \\
& & $10-13$ años & $11.9(2.1)$ \\
\hline
\end{tabular}

Nota: EP = Educación Primaria; ESO = Educación Secundaria Obligatoria 


\section{Procedimiento}

El cuestionario fue diseñado a partir de los nueve tipos de funciones ejecutivas considerados en el modelo teórico de partida (hiperactividad, impulsividad, memoria funcional, control emocional, planificación, organización, focalización de la atención, capacidad de concentración y flexibilidad cognitiva). Para tratar de garantizar la validez de contenido de la prueba, se elaboró un banco de 54 ítems, seis por cada factor hipotético, que fueron sometidos al juicio de seis expertos. Utilizando una tabla, cada experto calificó cada ítem por escrito, de acuerdo con dos criterios: su relevancia para medir el constructo y su claridad formal. Para ello, se utilizó una escala de 1 a 4 ( 1 = Nada; 2 = Poco; 3 $=$ Bastante $;=$ Totalmente).

A partir de la respuesta de los jueces, se calculó la puntuación promedio para cada ítem y el coeficiente de concordancia W de Kendall. A partir de estos resultados, se seleccionaron los tres ítems más valorados de cada factor hipotético. Los ítems seleccionados presentaron calificaciones promedio de entre 3.8 y 4 , con índices de concordancia superiores a .90 , tanto para claridad como para relevancia. Todos los enunciados finalmente utilizados son de elaboración propia, a excepción de los ítems 11, 23 y 25, que fueron tomados de la escala BRIEF (Gioia et al., 2000).

Una vez diseñado el cuestionario, se seleccionó una muestra piloto de familias para analizar su comprensión y propiedades psicométricas. Dado el objetivo exploratorio de esta primera fase de validación, se decidió acotar la muestra a los cursos $5^{\circ}$ y $6^{\circ}$ de Educación Primaria, por facilidad de acceso. Se contactó con los equipos directivos de dos centros escolares de Asturias, quienes fueron informados de los objetivos y del procedimiento del estudio, de su

carácter voluntario y anónimo y del tratamiento confidencial de los resultados. Todos dieron su consentimiento y trasladaron la petición de colaboración a las familias, que respondieron y devolvieron voluntariamente el cuestionario cumplimentado. El cuestionario fue contestado de manera anónima por las familias fuera del centro educativo, sin limitaciones temporales y contando con instrucciones detalladas en la primera página del cuestionario. Posteriormente, se aplicó la prueba a la muestra final, más amplia, siguiendo para ello el mismo procedimiento que el desarrollado con la muestra piloto.

\section{Análisis de datos}

Con los datos de la muestra piloto, se analizaron en primer lugar los ítems: posible presencia de enunciados no contestados por una parte apreciable de la muestra; índices de discriminación; $\alpha$ si se elimina el enunciado; normalidad y direccionalidad; y grado de correlación entre ítems. Se estudió, asimismo, la estructura factorial de la prueba mediante Análisis de Componentes Principales. Dada la correlación existente entre los factores, se utilizó Promax como método de rotación. Para realizar estos análisis se utilizó el paquete estadístico SPSS 21.0 .

Con los datos de la muestra final, se analizaron de nuevo los ítems, utilizando los mismos parámetros que con la muestra piloto. Posteriormente, se analizó la validez de constructo de la prueba mediante análisis factorial confirmatorio, con el programa Amos v21.0. Por último, siguiendo a autores como GarcíaFernández et al. (2016), se calcularon los pesos factoriales de los ítems en los factores y se evaluó la fiabilidad tanto del conjunto de la escala como de cada factor mediante el coeficiente $\alpha$ de Cronbach, indicador de su consistencia interna, con el paquete estadístico SPSS 21.0.

\section{Resultados}

\section{Muestra piloto}

Análisis de los ítems. Todas las categorías de respuesta, en cada ítem, fueron elegidas por un porcentaje de estudiantes; y sus desviaciones típicas mostraron valores entre 0.84 y 1.20 , lo cual es señal de que los ítems discriminan. La "correlación ítem-total corregida" es positiva en todos los ítems, con valores entre .428 y .699, lo que indica que todos contribuyen a medir lo que mide el test y además en la misma dirección. Si se elimina cualquiera de los enunciados, el $\alpha$ del conjunto de la escala disminuye. Ningún par de ítems ofrece un índice de correlación superior a .635 , lo que sugiere que el informante distingue el contenido de los diferentes enunciados. De acuerdo com los índices de asimetría y curtosis 
Tabla 2. Matriz de configuración y comunalidades de la Escala EFE-F tras la rotación, con la muestra piloto $(\mathrm{N}=209)$

\begin{tabular}{|c|c|c|c|}
\hline \multirow{2}{*}{ Ítems } & \multicolumn{2}{|c|}{ Factores } & \multirow{2}{*}{ Comunalidad } \\
\hline & AA & $\mathrm{AC}$ & \\
\hline 23. Cuando se le manda a por algo, se lo suele olvidar. & .835 & -.334 & .457 \\
\hline 3. Le cuesta organizarse sin control externo. & .763 & .028 & 610 \\
\hline 8. Le cuesta preparar la mochila para ir a clase. & .732 & -.227 & .377 \\
\hline 13. Su trabajo es poco cuidadoso. & .726 & -.007 & .520 \\
\hline 6. Comete errores por descuido en las tareas escolares o en otras actividades. & .717 & .073 & .585 \\
\hline 10. Le cuesta terminar lo que empieza. & .687 & .107 & .575 \\
\hline 19. Presta poca atención a los detalles. & .679 & .075 & .530 \\
\hline 18. Necesita ayuda para seguir realizando una tarea. & .630 & .179 & .571 \\
\hline 4. Cuando se le da más de una instrucción, recuerda sólo una de ellas. & .620 & -.037 & .357 \\
\hline 11. Tiene problemas para recordar cosas, incluso durante pocos minutos. & .615 & -.055 & .338 \\
\hline 22. Es incapaz de revisar los errores cuando realiza una actividad. & .605 & .186 & .543 \\
\hline 20. Empieza tareas o trabajos en el último minuto. & .604 & .173 & .526 \\
\hline 15. Se distrae con sus propios pensamientos. & .591 & 154 & .488 \\
\hline 12. Le cuesta estimar el tiempo necesario para realizar una tarea. & .433 & .337 & .485 \\
\hline 2. Es incapaz de cambiar su conducta ante diferentes situaciones. & .360 & .336 & .395 \\
\hline 21. Le cuesta adaptarse a nuevas situaciones. & .315 & .234 & .247 \\
\hline 17. Se enfada fácilmente. & -.256 & .936 & .640 \\
\hline 24. Es demasiado impulsivo/a en la forma de hablar o de comportarse. & -.167 & .894 & .638 \\
\hline 25. Los estallidos de ira o llanto son intensos, pero terminan rápidamente. & -.085 & .768 & .514 \\
\hline 14. Reacciona de forma más exagerada que los demás ante pequeñas cosas. & -.012 & .726 & .517 \\
\hline 16. Suele actuar sin pensar. & .029 & .722 & .549 \\
\hline 7. Le cuesta aceptar otros puntos de vista (en casa, en el juego, con las amistades). & .023 & .682 & .486 \\
\hline $\begin{array}{l}\text { 9. Interrumpe o se entromete en las actividades de los demás (en conversaciones, } \\
\text { juegos). }\end{array}$ & .037 & .652 & .457 \\
\hline 27. Le cuesta escuchar cuando se le habla. & .095 & .619 & .467 \\
\hline $\begin{array}{l}\text { 26. Actúa de modo más incontrolado que los demás (en fiestas de cumpleaños, } \\
\text { reuniones familiares, etc.). }\end{array}$ & .067 & .603 & .419 \\
\hline 5. Parece sentirse inquieto. & .115 & .590 & .447 \\
\hline 1. Le cuesta seguir las normas (en casa, en el juego). & .334 & .430 & .477 \\
\hline
\end{tabular}

Nota: AA = Autorregulación del Aprendizaje; $\mathrm{AC}=$ Autorregulación del Comportamiento

obtenidos y la prueba de Kolmogorov-Smirnov realizada, ninguno de los enunciados sigue una distribución normal. Todos los ítems muestran una asimetría positiva, lo que significa que los padres, madres o tutores tienden a marcar los valores bajos de la escala. Los índices de curtosis son negativos para las dos terceras partes de los ítems (18 de los 27), lo que indica que las puntuaciones tienden a agruparse menos y situarse por debajo de la curva de la distribución normal.

Análisis factorial exploratorio. El estudio de la estructura factorial del cuestionario se realizó a través de un primer análisis factorial de tipo exploratorio usando "Análisis de Componentes Principales" como método de extracción. La idoneidad de realizar análisis factoriales se confirmó calculando el índice de Kaiser-MeyerOlkin y la prueba de esfericidad de Bartlett $(\mathrm{KMO}=0.932 ;$ Chi-cuadrado=2916.618; $g l=351$; $p=.000$ ). El resultado de este primer análisis sugirió la existencia de dos factores, atendiendo a los resultados del gráfico de sedimentación y al contenido de los ítems que los componían.

Posteriormente se llevo a cabo un nuevo análisis factorial, forzando esta vez la retención a dos factores y usando de nuevo "Análisis de Componentes Principales" como método de extracción. Dada la interrelación existente entre los factores $(r=.631)$, se utilizo Promax como método de rotación. Como se puede observar en la Tabla 2, las comunalidades presentan valores por encima de .30 en todos los ítems, excepto en el 21. Los enunciados de la escala se agrupan en dos factores, que en su conjunto explican el $48.95 \%$ de la varianza. El factor Autorregulación del Aprendizaje explica un $40.75 \%$ de la varianza, e incluye ítems referidos a la emisión de conductas que manifiestan una carencia en la capacidad de atención (selectiva y sostenida), memoria, planificación y organización, vinculadas a situaciones académicas. El segundo factor, Autorregulación del Comportamiento, da cuenta del $8.2 \%$ de la varianza e incluye ítems referidos a 

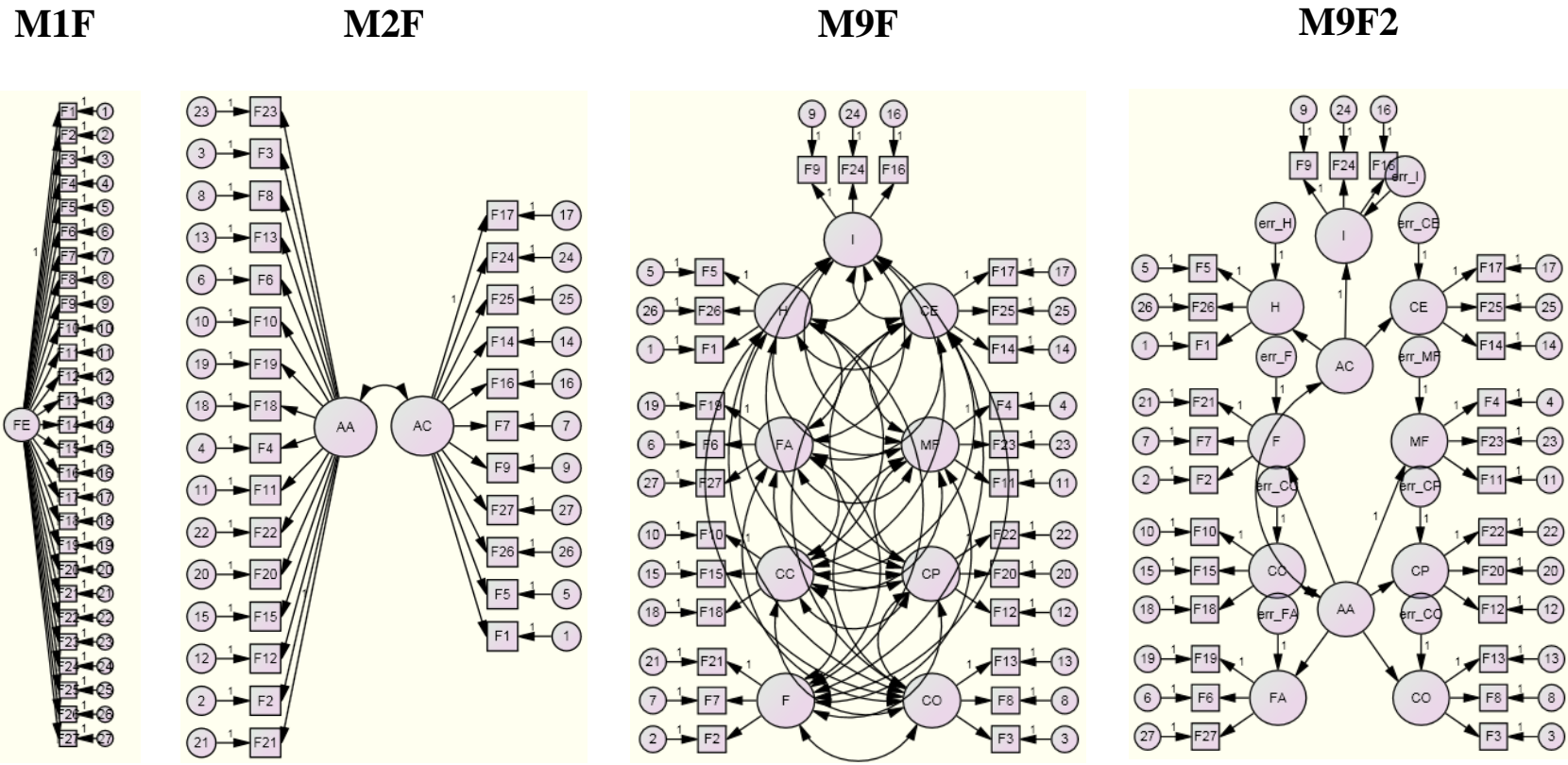

Figura 1. Estructuras factoriales de los modelos de un factor (M1F), de dos factores (M2F), de nueve factores (M9F), y de nueve factores con dos factores de segundo orden (M9F2)

la emisión de conductas impulsivas, a la falta de capacidad para inhibir la propia conducta y de controlar la expresión de sus emociones, vinculados al plano de lo social. Como se puede observar en la Tabla 2, todos los ítems que constituyen cada factor muestran pesos factoriales superiores a .30 .

\section{Muestra final}

Análisis de los ítems. Con la muestra final, todas las categorías de respuesta, en cada ítem, fueron elegidas por un porcentaje de estudiantes; y sus desviaciones típicas mostraron valores entre 0.84 y 1.25 , lo cual es señal de que los ítems discriminan. La "correlación ítem-total corregida" es positiva en todos los ítems, con valores entre .418 y .680 , lo que indica que todos contribuyen a medir lo que mide el test y además en la misma dirección. $\mathrm{Si}$ se elimina cualquiera de los enunciados, el $\alpha$ del conjunto de la escala disminuye. Ningún par de ítems ofrece un índice de correlación superior a .549, lo que sugiere que el informante distingue el contenido de los diferentes enunciados.

De acuerdo con los índices de asimetría y curtosis obtenidos y la prueba de KolmogorovSmirnov realizada, ninguno de los enunciados sigue una distribución normal. Todos los ítems muestran una asimetría positiva, lo que significa que los informantes (padres, madres o tutores) tienden a marcar los valores bajos de la escala. Respecto a la curtosis, los índices son negativos para la mayoría de los ítems (17 de los 27), lo que indica que las puntuaciones tienden a agruparse menos y a situarse por debajo de la curva de la distribución normal.

Análisis factorial confirmatorio. Para comprobar el ajuste del modelo obtenido con el análisis factorial exploratorio a los datos empíricos obtenidos con esta segunda muestra, más amplia, se realizaron análisis factoriales confirmatorios. Se comparó el grado de ajuste de este modelo de dos factores con el de otros tres modelos, también plausibles desde un punto de vista teórico (Figura 1). Partiendo de la base de que el cuestionario pretende evaluar el constructo "Funcionamiento ejecutivo", se ha puesto a prueba un Modelo de un factor (M1F), en el que cada ítem del cuestionario es explicado únicamente por ese único factor. El Modelo de dos factores (M2F) es el resultante del análisis factorial exploratorio realizado con la muestra piloto. Su estructura está compuesta por dos factores, interrelacionados significativamente entre sí: Autorregulación del Aprendizaje (AA) y Autorregulación del Comportamiento (AC). Cada ítem del cuestionario es explicado únicamente por un factor. El Modelo de nueve factores (M9F) se 
Tabla 3. Índices de bondad de ajuste de los modelos de un factor (M1F), dos factores (M2F), nueve factores (M9F) y nueve factores con dos factores de segundo orden (M9F2) de la Escala EFE-F, con el total de la muestra $(\mathrm{N}=1019)$

\begin{tabular}{lcccccccccc}
\hline Modelo & $\chi^{2}$ & $d f$ & $\chi^{2} / d f$ & $p$ & GFI & CFI & RMSEA & $($ LO90 & HI90 & $p)$ \\
\hline M1F & 2512.873 & 324 & 7.756 & .000 & .765 & .812 & .081 & .079 & .084 & .000 \\
M2F & 1386.659 & 323 & 4.293 & .000 & .900 & .908 & .057 & .054 & .060 & .000 \\
M9F & 1050.178 & 288 & 3.646 & .000 & .927 & .934 & .051 & .048 & .054 & .307 \\
M9F-2 & 1436.299 & 314 & 4.574 & .000 & .896 & .903 & .059 & .056 & .062 & .000
\end{tabular}

Nota: $\chi^{2}=$ Chi-cuadrado; $d f=$ Grados de libertad; $p=$ Nivel de probabilidad; $G F I=$ Goodness-of-Fit Index; $C F I=$ Comparative Fit Index; RMSEA = Root Mean Square Error of Approximation; LO90 y HI9O= Intervalos de confianza para evaluar la estimación del valor de RMSEA

Tabla 4. Pesos factoriales y fiabilidad de cada ítem y factor de la escala $(\mathrm{N}=1019)$

\begin{tabular}{|c|c|c|c|c|}
\hline Factor & Ítem & $\lambda$ & $\alpha$ & $R^{2}$ \\
\hline \multirow[t]{4}{*}{ Impulsividad } & & & .74 & \\
\hline & 9 & .64 & & .41 \\
\hline & 16 & .72 & & .52 \\
\hline & 24 & .75 & & .56 \\
\hline \multirow[t]{4}{*}{ Hiperactividad } & & & .67 & \\
\hline & 1 & .67 & & .44 \\
\hline & 5 & .64 & & .41 \\
\hline & 26 & .61 & & .37 \\
\hline \multirow[t]{4}{*}{ Control Emocional } & & & .74 & \\
\hline & 14 & .75 & & .57 \\
\hline & 17 & .73 & & .53 \\
\hline & 25 & .63 & & .40 \\
\hline \multirow[t]{4}{*}{ Memoria Funcional } & & & .75 & \\
\hline & 4 & .73 & & .54 \\
\hline & 11 & .72 & & .52 \\
\hline & 23 & .67 & & .45 \\
\hline \multirow[t]{4}{*}{ Capacidad de Planificación } & & & .69 & \\
\hline & 12 & .63 & & .39 \\
\hline & 20 & .65 & & .42 \\
\hline & 22 & .68 & & .46 \\
\hline \multirow[t]{4}{*}{ Capacidad de Organización } & & & .69 & \\
\hline & 3 & .69 & & .48 \\
\hline & 8 & .55 & & .30 \\
\hline & 13 & .71 & & .50 \\
\hline \multirow[t]{4}{*}{ Flexibilidad } & & & .61 & \\
\hline & 2 & .60 & & .37 \\
\hline & 7 & .66 & & .43 \\
\hline & 21 & .49 & & .24 \\
\hline \multirow[t]{4}{*}{ Capacidad de Concentración } & & & .71 & \\
\hline & 10 & .73 & & .53 \\
\hline & 15 & .61 & & .38 \\
\hline & 18 & .68 & & .47 \\
\hline \multirow[t]{4}{*}{ Focalización de la Atención } & & & .69 & \\
\hline & 6 & .64 & & .41 \\
\hline & 19 & .64 & & .41 \\
\hline & 27 & .66 & & .44 \\
\hline
\end{tabular}

corresponde con el modelo teórico de partida. Su estructura está representada por nueve factores, interrelacionados significativamente entre sí (Impulsividad, Hiperactividad, Control Emocional, Memoria Funcional, Capacidad de Planificación, Capacidad de Organización, Flexibilidad,
Capacidad de Concentración y Focalización de la Atención) y cada ítem del cuestionario es explicado únicamente por un factor. El Modelo de nueve factores con dos factores de segundo orden (M9F2) trata de combinar el modelo teórico inicial con el resultado del análisis factorial 
exploratorio. Es igual que el M9F pero en él se postula que las relaciones entre los nueve factores de primer orden indican la existencia de dos factores de segundo orden (Autorregulación del Aprendizaje y Autorregulación del Comportamiento), que explican significativamente los factores de primer orden.

Los resultados obtenidos (Tabla 3) indican que el M9F es, de los cuatro modelos comparados, el que mejor bondad de ajuste ofrece. El M9F presenta los índices GFI y CFI más altos, así como los índices $\chi^{2} / d f$ y RMSEA más bajos, de los cuatro modelos puestos a prueba.

Pesos factoriales y análisis de fiabilidad. Los pesos factoriales de los ítems en los factores presentan valores en general altos (Tabla 4). Todos los ítems, excepto dos, presentan un coeficiente de regresión estandarizado alto $(\lambda>$.60). Ninguno presentó niveles menores a .40 . El $\alpha$ de Cronbach (consistencia interna) es alto para el conjunto del cuestionario (.94) y moderado para los factores. En general, la fiabilidad de los ítems (R2) tiende a ser moderada. La proporción de varianza en el ítem explicada por la variable latente se sitúa entre el $24 \%$ y el $57 \%$.

\section{Discusión}

El objetivo de este trabajo ha sido presentar un cuestionario breve de calificación de conducta para la evaluación del funcionamiento ejecutivo en niños y adolescentes de 7 a 18 años, basada en la información proporcionada por sus familias, así como sus propiedades psicométricas tras su aplicación a una amplia muestra de familias de Asturias (España). A la vista de los resultados obtenidos con la muestra final, la Escala de Funcionamiento Ejecutivo para Familias (Escala $E F E-F)$ ha mostrado unas adecuadas propiedades psicométricas para ser utilizada con el objetivo con el que fue creada: identificar posibles déficits en las diferentes funciones ejecutivas evaluadas, a partir de indicadores conductuales en situaciones naturales.

Los análisis factoriales confirmatorios realizados con la muestra final han revelado una adecuada validez de constructo de la prueba. El Modelo de 9 factores ofrece unos índices de ajuste que, sin llegar a ser excelentes de acuerdo con los criterios más estrictos (Ruíz, Pardo, \& San Martín, 2010), se sitúan dentro de los límites comúnmente considerados como aceptables (Gil-Monte \& Zúñiga-Caballero, 2010; Piemontesi, Heredia, \& Furlan, 2012). En todo caso, sus indicadores de ajuste son mejores que los obtenidos con los otros tres modelos puestos a prueba, también plausibles desde un punto de vista teórico. Los pesos factoriales de cada ítem en los factores son en general altos, así como los coeficientes de regresión estandarizados. La fiabilidad de la prueba, analizada en términos de consistencia interna, fue alta para la escala completa y moderada para cada uno de sus nueve factores, aunque razonable teniendo en cuenta el escaso número de ítems que los componen (tres por factor).

La principal implicación teórica de este estudio radica en que los resultados obtenidos en la muestra final apoyan la solidez del marco teórico de partida. El modelo que mejor ajuste a los datos ha mostrado ha sido el modelo inicialmente hipotetizado, constituido por nueve factores. Este resultado reafirma la hipótesis de la naturaleza multidimensional del constructo funcionamiento ejecutivo, el cual parece estar compuesto de diferentes funciones ejecutivas, en consonancia con estudios previos (Egeland \& Fallmyr, 2010; Huizinga \& Smidts, 2011; Korzeniowski, 2011; Roselli et al., 2008).

Desde un punto de vista práctico, la Escala $E F E-F$ supone una contribución para la identificación de niños o adolescentes con alteraciones en las funciones ejecutivas, con fines clínicos o de investigación. En contextos clínicos, la Escala EFE-F contribuirá a un diagnóstico preciso y, con ello, a un tratamiento ajustado. En contextos de investigación, aportará información relevante sobre la naturaleza de las funciones ejecutivas, así como sobre la prevalencia, incidencia y comorbilidad de los déficits ejecutivos con otras problemáticas. Como cuestionario de calificación de la conducta que es, permite complementar la información obtenida con medidas neuropsicológicas basadas en la ejecución, al referirse a conductas emitidas en situaciones naturales cotidianas como el hogar o la escuela. Adicionalmente, presenta algunas ventajas frente a otros cuestionarios de este tipo previamente publicados. En primer lugar, se trata 
de una escala breve, lo que favorece la respuesta de las familias. Esto resulta especialmente interesante en entornos clínicos en los que las familias deban cumplimentar numerosos informes o en entornos de investigación que se necesite obtener una muestra suficiente y representativa de la población analizada. En segundo lugar, se trata de una prueba que abarca el amplio rango de edad comprendido entre los 7 y 18 años, lo que resulta especialmente relevante debido a la existencia de numerosos trastornos o dificultades de aprendizaje durante la infancia y adolescencia en los que el funcionamiento ejecutivo se ve comprometido. Por último, la Escala EFE-F ha sido validada en población española, lo que añade garantías para su aplicación en nuestro entorno cultural.

No obstante, además de estas aportaciones, la Escala EFE-F presenta ciertas limitaciones. En primer lugar, no se ha utilizado un procedimiento de selección de la muestra que garantice su representatividad respecto a la población a la que se pretendía generalizar los resultados y el uso de la escala. Por ello, este debe ser un aspecto a mejorar en futuras aplicaciones de la prueba. En segundo lugar, el ajuste de los datos al modelo de nueve factores, si bien entra dentro de los niveles de ajuste considerados comúnmente como aceptables, aún resulta mejorable. En tercer lugar, la consistencia interna de ciertos factores es algo baja, aunque razonable de acuerdo con su escaso número de ítems. Dado que se pretende desarrollar una escala lo más breve posible, se debería descartar de cara al futuro la opción de aumentar artificialmente su longitud, tratando en su lugar de encontrar algún ítem que represente mejor el contenido del factor. Se espera que esto incida, a su vez, en el nivel de ajuste del modelo. Por último, es necesario destacar la necesidad de emplear este tipo de medidas basadas en observación como complemento a otro tipo de instrumentos previamente validados, como son las medidas basadas en la ejecución del individuo. Si bien el uso de escalas de calificación de la conducta contribuye a añadir validez ecológica a la evaluación de las funciones ejecutivas, ciertas dificultades relacionadas con la administración de este tipo de instrumentos, como la deseabilidad social o la presencia de sesgos perceptivos en el informante, pueden ser controlados con la administración conjunta de múltiples medidas de evaluación.

\section{Referencias}

Baddeley, A. D. (2000). The episodic buffer: A new component of working memory? Trends in Cognitive Sciences, 4, 417-423. doi: 10.1016/S1364-6613(00)01538-2

Baddeley, A. D. (2007). Working Memory, Thought, and Action. Oxford: Oxford University Press.

Barkley, R. A. (1997). ADHD and the nature of self-control. New York: Guilford

Barkley, R. A. (1998). Attention deficit hyperactivity disorders: A handbook for diagnosis and treatment. New York: Guilford Press.

Borys, S. V., Spitz, H. H., \& Dorans, B. A. (1982). Tower of Hanoi performance of retarded young adults and nonretarded children as a function of solution length and goal state. Journal of Experimental Child Psychology, 33, 87-110. doi: 10.1016/0022-0965(82)900 08-X

Brock, L. L., Rimm-Kaufman, S. E., Nathanson, L., \& Grimm, K. J. (2009). The contribution of 'hot' and 'cool' executive function to children's academic achievement and classroom behavior. Early Childhood Research Quarterly, 24, 337-349. doi: 10.1016/j.ecresq.2009.06.001

Collins, A., \& Koechlin, E. (2012). Reasoning, learning, and creativity: Frontal lobe function and human decision making. PLoS Biology, 10, e1001293. doi: 10.1371/journal.pbio.100 1293

Cragg, L., \& Gilmore, C. (2014). Skills underlying mathematics: The role of executive function in the development of mathematics proficiency. Trends in Neuroscience and Education, 3(2), 63-68. doi: 10.1016/j.tine.2013.12.001

Craig, F., Margari, F., Legrottaglie, A. R., Palumbi, R., de Giambattista, C., \& Margari, L. (2016). A review of executive function deficits in autism spectrum disorder and attention-deficit hyperactivity disorder. Neuropsychiatric Disease and Treatment, 12, 1191-1202. doi:10.2147/NDT.S104620 
Delis, D. C., Kaplan, E. y Kramer, J. H. (2001). Delis-Kaplan Executive Function System. San Antonio: The Psychological Corporation.

Di Trani, M., Casini, M. P., Capuzzo, F., Gentile, S., Bianco, G., Menghini, D., \& Vicari, S. (2011). Executive and intellectual functions in attention-deficit/hyperactivity disorder with and without comorbidity. Brain and Development, 33(6), 462-469. doi: 10.1016/j.braindev.2010.06.002.

Diamond, A. (2013). Executive functions. Annual Review of Psychology, 64, 135-68. doi: 10.1146/annurev-psych-113011-143750

Egeland, J. y Fallmyr, O. (2010). Confirmatory factor analysis of the Behavior Rating Inventory of Executive Function (BRIEF): Support for a distinction between emotional and behavioral regulation. Child Neuropsychology, 16(4), 326-337. doi: 10.1080/09297041003601462

Elosua, P., Mujika, J., Almeida, L. S., \& Hermosilla, D. (2014). Procedimientos analítico-racionales en la adaptación de tests. Adaptación al español de la batería de pruebas de razonamiento. Revista Latinoamericana de Psicología, 46(2), 117-126. doi: 10.1016/S0120-0534(14)70015-9

García, T., González-Pienda, J. A., Rodríguez, C., Álvarez-García, D., \& Álvarez, L. (2014). Psychometric characteristics of the BRIEF scale for the assessment of executive functions in Spanish clinical population. Psicothema, 26(1), 47-52. doi: 10.7334/psicothema2013.149

García, T., Kroesbergen, E. H., Rodríguez, C., González-Castro, P., \& Gonzalez-Pienda, J. A. (2015). Factors involved in making postperformance judgments in mathematics problem-solving. Psicothema, 27(4), 374-380. doi: $10.7334 /$ psicothema2015.25

García, T., Rodríguez, C., González-Castro, P. Álvarez, D., Cueli, M., \& González-Pienda, J.A. (2013). Funciones ejecutivas en niños y adolescentes con trastorno por déficit de atención con hiperactividad y dificultades lectoras. International Journal of Psychology \& Psychological Therapy, 13(2), 179-194.

García-Fernández, J. M., Inglés-Saura, C. J., Vicent-Juan, M., Gonzálvez-Maciá, C., PérezSánchez, A. M., \& San Martín, N. L. (2016).
Validación de la Escala de Autoeficacia Percibida Específica de Situaciones Académicas en Chile y su Relación con las estrategias de aprendizaje. Revista Iberoamericana de Diagnóstico y Evaluación - e Avaliação Psicológica, 1, 118-131.

Garon, N., Bryson, S. E., \& Smith, I. M. (2008). Executive function in preschoolers: A review using an integrative framework. Psychological Bulletin, 134, 31-60. doi: 10.1037/0033-2909.134.1.31

Gil-Monte, P. R., \& Zúñiga-Caballero, L. C. (2010). Validez factorial del "Cuestionario para la Evaluación del Síndrome de Quemarse por el Trabajo" (CESQT) en una muestra de médicos mexicanos. Universitas Psychologica, 9(1), 169-178.

Gioia, G. A., Isquith, P. K., Guy, S., \& Kenworthy, L. (2000). BRIEF: Behavior Rating Inventory of Executive Function professional manual. Lutz, FL: Psychological Assessment Resources, Inc.

Golden, C. J. (1974). Effect of differing number of colors on the Stroop Color and Word Test. Perceptual and Motor Skills, 39, 1067-1070. doi: 10.2466/pms.1974.39.1.550

Goulden, L., \& Silver, C.H. (2009). Concordance of the Children's Executive Functions Scale with established tests and parent rating scales. Journal of Psychoeducational Assessment, 20, 1-13. doi: 10.1177/0734282909335574.

Heaton, R. K., Chelune, G. J., Talley, J. L., Kay, G. G., \& Curtis, G. (1993). Wisconsin Card Sorting Test (WCST). Manual revised and expanded. Odessa: Psychological Assessment Resources Inc.

Holmes, J., Gathercole, S. E., Place, M., Alloway, T. P., Elliott, J. G., \& Hilton, K. A. (2010). The diagnostic utility of executive function assessments in the identification of ADHD in children. Child and Adolescent Mental Health, 15(1), 37-43. doi: 10.1111/j.1475-3588.2009 $.00536 . \mathrm{x}$

Huizinga, M., \& Smidts, D. P. (2011). Age-related changes in executive function: A normative study with the Dutch version of the Behavior Rating Inventory of Executive Function (BRIEF). Child Neuropsychology, 17(1), 5166. doi: 10.1080/09297049.2010.509715.

Kobayashi, H., \& Kobayashi, H. (2007). Study of 
the behavioural assessment of the dysexecutive syndrome (BADS) in schoolaged children. Journal of Saitama University, 56, 49-57.

Korzeniowski, C. G. (2011). Desarrollo evolutivo del funcionamiento ejecutivo y su relación con el aprendizaje escolar. Revista de Psicología, 7(13), 7-26.

Leung, R. C., Vogan, V. M., Powell, T. L., Anagnostou, E., \& Taylor, M. J. (2016). The role of executive functions in social impairment in autism spectrum disorder. Child Neuropsychology, 22(3), 336-344. doi: 10.1080/09297049.2015.1005066

Lezak, M. D. (2004). Neuropsychological assessment. New York: Oxford University Press.

Lezak, M., Howieson, D., Bigler, E., \& Tranel, D. (2012). Neuropsychological assessment $\left(5^{\text {th }}\right.$ ed.). New York: Oxford University Press.

Lozano, A., \& Ostrosky, F. (2011). Desarrollo de las funciones ejecutivas y de la corteza prefrontal. Revista Neuropsicología, Neuropsiquiatría y Neurociencias, 11(1), 159172.

Martínez, R. (1995). Psicometría: Teoría de los tests psicológicos y educativos. Madrid: Síntesis.

Mayoral, S., Roca, M., Timoneda, C., \& Serra, M. (2015). Mejora de la capacidad de planificación cognitiva del alumnado de primer curso de Educación Secundaria Obligatoria. Aula Abierta, 43(1), 9-17. doi: 10.1016/j.aula.2014.10.001

Miyake, A., Friedman, N. P., Emerson, M. J., Witzki, A. H., Howerter, A., \& Wager, T. D. (2000). The unity and diversity of executive functions and their contributions to complex "frontal lobe" tasks: a latent variable analysis. Cognitive Psychology, 41, 49-100. doi: 10.1006/cogp.1999.0734

Musso, M (2009). Evaluación de funciones ejecutivas en niños: Análisis y adaptación de pruebas en un contexto escolar. Revista Iberoamericana de Diagnóstico y Evaluación - e Avaliação Psicológica,1(27), 157-178.

Piemontesi, S. E., Heredia, D. E., \& Furlan, L. A. (2012). Propiedades psicométricas de la versión en español revisada del Inventario Alemán de Ansiedad ante Exámenes (GTAI-
AR) en universitarios argentinos. Universitas Psychologica, 11(1), 177-186.

Portellano, J. A., Martínez-Arias, R., \& Zumárraga, L. (2009). ENFEN: Evaluación neuropsicológica de las funciones ejecutivas en niños. Madrid: TEA Ediciones.

Roselli, M., Jurado, M. B., \& Matute, E. (2008). Las funciones ejecutivas a través de la vida. Revista Neuropsicología, Neuropsiquiatría y Neurociencias, 8(1), 23-46.

Ruíz, M. A., Pardo, A., \& San Martin, R. (2010). Modelos de Ecuaciones Estructurales. Papeles del Psicólogo, 31(1), 34-45.

Shimoni, M., Engel-Yeger, B., \& Tirosh, E. (2012). Executive dysfunctions among boys with Attention Deficit Hyperactivity Disorder (ADHD):Performance-based test and parents report. Research in Developmental Disabilities, 33(3), 858-65. doi: 10.1016/j.ridd.2011.12.014.

Silver, C. H., Kolitz-Russell, S., Bordini, F., \& Fairbanks, J. (1993). The Children's Executive Functions Scale. Unpublished rating scale.

Stroop, J. R. (1935). Studies of interference in serial verbal reactions. Journal of Experimental Psychology, 18, 643-662. doi: 10.1037/h0054651

Thorell, L. B., \& Nyberg, L. (2008). The Childhood Executive Functioning Inventory (CHEXI): A new rating instrument for parents and teachers. Developmental Neuropsychology, 33, 536-552. doi: 10.1080/87565640802101516

Tirapú-Ustárroz, J., García, A., Luna, P., Roig, T., \& Pelegrín, C. (2008). Modelos de funciones y control ejecutivo (II). Revista de Neurología, 46, 742-750.

Verdejo-García, A., \& Bechara, A. (2010). Neuropsicología de las funciones ejecutivas. Psicothema, 22(2), 227-235. 